\title{
Relationship Between Biopsy Core $\alpha$-Methylacyl-CoA Racemase Positivity and Five-Year Biochemical Recurrence in D'Amico Low- and Intermediate-Risk Prostate Cancer
}

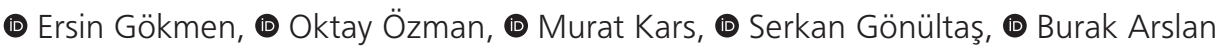 \\ University of Health Sciences Turkey, Gaziosmanpaşa Training and Research Hospital, Clinic of Urology, Istanbul, Turkey
}

\begin{abstract}
Objective: This study aims to explore the association between alpha methylacyl A coenzyme racemase (AMACR)/P504S staining intensity of prostate biopsy cores and five-year biochemical recurrence after radical prostatectomy in patients diagnosed with localised prostate cancer.

Materials and Methods: Patients who underwent radical prostatectomy for organ-limited prostate cancer were retrospectively examined. Twenty-five patients without recurrence after definitive treatment and 25 patients with prostate-specific antigen (PSA) recurrence at postoperative follow-up were classified as group 1 and group 2. Positive prostate biopsy cores of patients were stained with AMACR/P504S, prospectively. Staining intensities were scored as negative (score=0), weak (score=1), moderate (score=2) and strong (score=3). Groups were compared regarding AMACR/P504S staining intensities of biopsy cores.

Results: The mean AMACR/P504S staining scores of positive biopsy cores were $1.88 \pm 0.85$ and $1.27 \pm 1.22$ for group 1 and group 2 . There was a statistically significant relationship between mean AMACR/P504S staining scores and PSA recurrence $(p=0.002)$. AMACR score groups were not separated concerning biochemical recurrence endpoints in the Kaplan-Meier analysis $(p=0.43)$.

Conclusion: There is a significant relationship between increased AMACR/P504S expression in cancerous prostate tissue and PSA recurrence after radical prostatectomy.

Keywords: AMACR/P504S, PSA, prostate cancer
\end{abstract}

\section{Introduction}

Prostate cancer is the second most common cancer in men worldwide, according to current data of the GLOBOCAN study (1). It is the fifth most common cause of cancer-related death. Relatively low mortality compared with incidence rates of prostate cancer has been attributed to the widespread use of prostate-specific antigen (PSA) as a screening tool, early diagnosis of patients, and cured at the localised early stage. Especially since the mid-1980s, substantial improvements have been made in the diagnosis and treatment of prostate cancer.

$\alpha$-Methylacyl-CoA Racemase/P504S (AMACR/P504S) is a cytoplasmic immune marker protein found by $\mathrm{Xu}$ and colleagues in 2000 (2). It was obtained by high throughput microarray imaging and CDNA library subtraction analysis from prostate tissue. AMACR is mainly localised in peroxisomes in prostate cancer cells. However, its up-regulation causes cancer to start and progress in some cells due to DNA oxidative damage and other unknown causes (3).
Serum PSA levels after radical prostatectomy should be too low to be measured. An increase in serum PSA levels after primary local treatment, or biochemical recurrence, was defined as an early and the first indicator of inadequate treatment. After curative treatment, $20 \%$ to $40 \%$ of patients develop biochemical recurrence within 10 years (4). Forty-five per cent of biochemical recurrences occur within two years, $77 \%$ occur within the first five years, and only $23 \%$ occur after five years.

AMACR's effectiveness has been the subject of research in many disciplines, from point-of-care prostate cancer diagnosis to molecular imaging of cancer $(5,6)$. Also, AMACR is a promising molecule to predict biochemical recurrence (7). This study aims to explore the association between AMACR/P504S staining intensity of prostate biopsy cores and the five-year biochemical recurrence after radical prostatectomy in patients diagnosed with localised prostate cancer.

Cite this article as: Gökmen E, Özman O, Kars M, Gönültaş S, Arslan B. Relationship Between Biopsy Core $\alpha$-Methylacyl-CoA Racemase Positivity and Five-Year Biochemical Recurrence in D'Amico Low- and Intermediate-Risk Prostate Cancer. Bull Urooncol 2021;20(2):92-95

Address for Correspondence: Ersin Gökmen, University of Health Sciences Turkey, Gaziosmanpaşa Training and Research Hospital, Clinic of Urology, İstanbul, Turkey Phone: +90 2129453000 E-mail: drersingokmen@gmail.com ORCID-ID: orcid.org/0000-0002-9845-163X 


\section{Materials and Methods}

Patients who underwent radical prostatectomy for D'Amico low- and intermediate-risk prostate cancer were retrospectively examined. Patients who have preoperative PSA $>20 \mathrm{ng} / \mathrm{mL}$, clinical or pathological stage T2c and above, positive lymph node metastasis and biopsy Gleason score $>7$ [International Society of Urological Pathology (ISUP) grade group 4-5] were excluded from the study. Fifty patients who had no surgical margin and lymph node positivity were included in the study. Twenty-five patients who did not develop a biochemical recurrence within at least five years of postoperative follow-up and 25 patients with PSA recurrence were classified as group 1 and group 2, respectively. PSA values $\geq 0.2 \mathrm{ng} / \mathrm{mL}$ at postoperative follow-up were considered biochemical recurrences.

Patients' positive prostate biopsy cores were stained with AMACR/P504S, prospectively. The immunohistochemical staining technique was performed as in the similar study we conducted earlier (8). Staining intensities were scored as negative $($ score $=0)$, weak (score $=1)$, moderate (score $=2$ ) and strong (score=3) (Table 1 and Figure 1) (9). Among the same patient's positive cores, the most intensely stained core's score was determined as the relevant patient's AMACR staining score. The biopsy Gleason score of four patients reported as $5(3+2)$ was changed to $6(3+3)$ according to the updated scoring system.

\begin{tabular}{|l|l|}
\hline Table 1. Staining classification of prostate biopsy cores \\
\hline Score & AMACR/P504S Staining \\
\hline 0 & Negative staining \\
\hline 1 & Weak focal staining \\
\hline 2 & Moderate cytoplasmic staining \\
\hline 3 & Diffuse cytoplasmic staining \\
\hline AMACR: Alpha methylacyl A coenzyme racemase \\
\hline
\end{tabular}

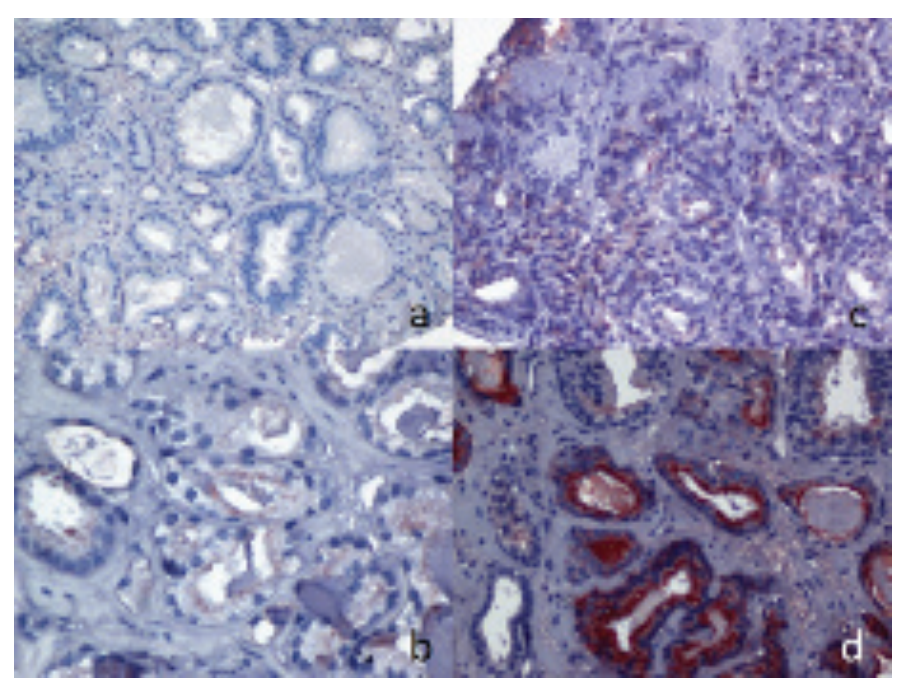

Figure 1. (a) Negative staining (AMACR/P504S X 200). (b) Weak, focal apical granular staining (AMACR/P504S X 600). (c) Moderate, disseminated cytoplasmic staining (AMACR/P504S X 400). (d) Diffuse, strong cytoplasmic staining (AMACR/P504S X 400)

AMACR: Alpha methylacyl A coenzyme racemase

\section{Statistical Analysis}

The variables' normality was checked by the KolmogorovSmirnov test. Groups were compared regarding AMACR/ P504S staining intensities of biopsy cores, positive core number (Mann-Whitney U test), age, preoperative prostate volume and PSA value, tumour volume percentage (Student t-test), biopsy and radical prostatectomy Gleason scores/ISUP grades, up and down-grading rates (chi-square test). In addition, patients were divided into three subgroups according to AMACR staining intensity scores, and the Kaplan-Meier test was applied to each subgroup. A $p<0.05$ value was considered statistically significant.

\section{Results}

The mean follow-up time was 40.48 (12-63) months when recurrent patients were included in the study. The patients' mean age in the PSA recurrence group was slightly higher than the other group ( $66.1 \pm 6.2$ vs $63.5 \pm 7.9$ years, respectively). However, the difference between ages was not statistically significant $(p=0.25)$. There was a statistically significant difference between the two groups regarding preoperative PSA values $(9.4 \pm 5.5$ vs $14.7 \pm 7.1 \mathrm{ng} / \mathrm{dL}, \mathrm{p}=0.004)$. There was no statistically significant difference between the groups' preoperative prostate volumes (47.2 \pm 17.9 vs $54.6 \pm 20.3 \mathrm{~mL}, \mathrm{p}=0.36$ ). The distribution of the patients' biopsy Gleason/ISUP grade group is shown in Table 2. No statistically significant difference was found between the grade distributions $(p=0.55)$. The positive core median was three in both groups $(p=0.65)$. The mean AMACR/P504S staining scores of positive biopsy cores were $1.88 \pm 0.85$ and $1.27 \pm 1.22$ for group 1 and group 2, respectively. The AMACR/ P504S staining score of positive cores were significantly higher in patients with PSA recurrence than non-recurrent patients $(p=0.002)$.

\begin{tabular}{|c|c|c|c|}
\hline & $\begin{array}{l}\text { PSA recurrence + } \\
\text { Group } 1\end{array}$ & $\begin{array}{l}\text { PSA recurrence - } \\
\text { Group } 2\end{array}$ & p-value \\
\hline Patient number & 25 & 25 & \\
\hline Age (year, mean) & $66.1 \pm 6.2$ & $63.5 \pm 7.9$ & $0.25^{*}$ \\
\hline (median, range) & $67(54-76)$ & $65.5(44-75)$ & \\
\hline $\begin{array}{l}\text { Preop. PSA (ng/mL, } \\
\text { mean) }\end{array}$ & $14.7 \pm 7.1$ & $9.4 \pm 5.5$ & $0.004^{*}$ \\
\hline \multicolumn{4}{|c|}{ Biospy Gleason scores/ISUP grades } \\
\hline Gleason 3+3/ISUP 1 & $21 / 25(84 \%)$ & $18 / 25(72 \%)$ & \\
\hline Gleason 3+4/ISUP 2 & $4 / 25(16 \%)$ & $4 / 25(16 \%)$ & $0.55^{\alpha}$ \\
\hline Gleason 4+3/ISUP 3 & $0 / 25(0 \%)$ & $3 / 25(12 \%)$ & \\
\hline $\begin{array}{l}\text { Positive core number } \\
\text { (median) }\end{array}$ & $3(1-6)$ & $3(1-8)$ & $0.65^{\beta}$ \\
\hline $\begin{array}{l}\text { TRUS prostate } \\
\text { volume (mL, mean) } \\
\end{array}$ & $47.2 \pm 17.9$ & $54.6 \pm 20.3$ & $0.36^{*}$ \\
\hline $\begin{array}{l}\text { Mean AMACR/P504S } \\
\text { staining score of } \\
\text { positive cores }\end{array}$ & $1.88 \pm 0.85$ & $1.27 \pm 1.22$ & $0.002^{\beta}$ \\
\hline
\end{tabular}




\begin{tabular}{|c|c|c|c|}
\hline & $\begin{array}{l}\text { PSA Recurrence + } \\
\text { Group } 1\end{array}$ & $\begin{array}{l}\text { PSA Recurrence - } \\
\text { Group } 2\end{array}$ & $p$-value \\
\hline Patient number & 25 & 25 & \\
\hline \multicolumn{4}{|c|}{ RP Gleason scores/ISUP grades } \\
\hline Gleason 3+3/ISUP 1 & $17 / 25(68 \%)$ & $18 / 25(72 \%)$ & \\
\hline Gleason 3+4/ISUP 2 & $5 / 25(20 \%)$ & $2 / 25(8 \%)$ & $0.37^{\alpha}$ \\
\hline Gleason 4+3/ISUP 3 & $3 / 25(12 \%)$ & $5 / 25(20 \%)$ & \\
\hline Up-grading rate & $8 / 25(32 \%)$ & $5 / 25(20 \%)$ & $0.52^{\alpha}$ \\
\hline Down-grading rate & $1 / 25(4 \%)$ & $3 / 25(12 \%)$ & $0.61^{\alpha}$ \\
\hline $\begin{array}{l}\text { Tumor volume } \\
\text { percentage (mean) }\end{array}$ & $32.1 \pm 23.8$ & $25.4 \pm 15.1$ & $0.67^{*}$ \\
\hline
\end{tabular}

${ }^{a}$ chi-square test, *Student t-test, RP: Radical prostatectomy, PSA: Prostate-specific antigen, ISUP: International Society of Urological Pathology

The distribution of the patients' radical prostatectomy Gleason/ ISUP grade group is shown in Table 3. No statistically significant difference was found between the grade distributions $(p=0.37)$. Gleason/ISUP up-grading was detected in $32 \%(8 / 25)$ of patients in group 1 and in $20 \%(5 / 25)$ of patients in group 2 $(p=0.52)$. Gleason/ISUP down-grading was detected in $4 \%$ $(1 / 25)$ of patients in group 1 and in $12 \%(3 / 25)$ of patients in group $2(p=0.61)$. There was no difference in the tumour volume percentage between the groups $(32.1 \pm 23.8$ vs $25.4 \pm 15.1$, $\mathrm{p}=0.67$ ).

The patients were divided into three subgroups according to their biopsy AMACR scores. A Kaplan-Meier test was performed according to the biochemical recurrence outcome (Graphic 1). A test of equality of recurrence distributions for the different AMACR scores did not show statistical significance $(p=0.43)$.

\section{Discussion}

Our study's main finding showed a significant relationship between the increased biopsy core AMACR staining intensity and biochemical recurrence after radical prostatectomy. This clinical finding provides indirect evidence of a possible relationship between prostate cancer aggressiveness and increased AMACR cancer cell expression. However, the AMACR score groups were not separated regarding biochemical recurrence endpoints in the Kaplan-Meier analysis.

Box and colleagues (10) described the relationship between AMACR and biochemical recurrence as marginal in a study of 218 patients who underwent radical prostatectomy for localised prostate cancer. On the other hand, an early study investigating the relationship between AMACR-prostate cancer and lower AMACR tissue expression has been associated with an increased rate of biochemical recurrence (7). Inconsistent results regarding the relationship between AMACR and worse prostate cancer outcomes can be explained by varying AMACR expression during prostate cancer's natural course. Studies show that AMACR expression increases in cancerous cells compared with benign prostate cells but decreases as cancer cells' differentiation decreases. Luo et al. (11) demonstrated in 2002 that the AMACR gene is up-regulated in prostate cancer.
A growing body of literature suggests that the gene groups' predictive value may be more effective than clinical and pathological parameters, such as PSA and Gleason score. Overexpression of four genes, including AMACR, was shown to have a significant relationship with aggressive disease characteristics, such as extracapsular extension, tumour stage, and seminal vesicle invasion in a study conducted in 2019 (12). This statistically significant relationship showed better overall clinical performance than PSA and Gleason score. The AMACR score showed a better diagnostic value than serum PSA in another recent study (13). In that study, AMACR and PSA messenger RNA (mRNAs) obtained by urine sediment analysis were evaluated by quantitative real-time polymerase chain reaction. In our study, the PSA and biopsy core AMACR staining intensity differences between the groups regarding biochemical recurrence after radical prostatectomy were statistically significant.

Since AMACR expression is directly related to carcinogenesis, it makes the AMACR molecule a parameter that offers different clinical benefits than PSA. It is known that PSA synthesis in the prostate cell does not increase significantly even in neoplastic processes (14). The increase in serum PSA is an indirect indicator of the increase in the cancerous cell number, deterioration of the intercellular connections and the basement membrane (15). AMACR's presence in serum has been demonstrated, but no statistically significant difference was found between serum AMACR levels in patients with and without prostate cancer (16). Moreover, AMACR is not a prostate-specific molecule (17). The above-mentioned disadvantages of the molecule in the systemic circulation overshadow its superiority against PSA in the cancer microenvironment. This causes the molecule to be a parameter dependent on tissue diagnosis and limits its clinical use as a candidate for prostate cancer marker. In this context, seminal fluid studies are far from providing the expected results (18).

\section{Study Limitations}

Our study has some limitations. Retrospective patient data and material collection, excluding patients with insufficient data, has affected the groups' random distribution. In addition, the number of patients included in the study was below the number obtained by power analysis due to the lack of staining kits. The categorical evaluation of AMACR staining intensity restricted the statistical efficiency of the parameter. Further scoring can be developed, like the Gleason score, which considers the overall biopsy core specimen. In addition, high up-grading rates (group 1: $32 \%$ vs group 2: $20 \%$ ) in both groups weakened the relationship between the biopsy findings and the clinical course of patients after radical prostatectomy.

\section{Conclusion}

There is a significant relationship between increased AMACR/ P504S expression in cancerous prostate tissue and PSA recurrence after radical prostatectomy. Prospective clinical studies are needed to demonstrate AMACR's predictive value of biochemical recurrence with a high level of evidence.

\section{Acknowledgements}

Publication: The results of the study were not published in full or in part in form of abstracts. 
Contribution: There is not any contributors who may not be listed as authors.

Conflict of Interest: No conflict of interest was declared by the authors.

Financial Disclosure: The authors declared that this study received no financial support.

\section{Ethics}

Ethics Committee Approval: Retrospective study.

Informed Consent: Retrospective study.

Peer-review: Externally peer-reviewed.

\section{Authorship Contributions}

Concept: E.G., Design: E.G., Data Collection or Processing: E.G., M.K., Analysis or Interpretation: E.G., O.Ö., B.A., Literature Search: S.G., Writing: E.G., M.K., B.A.

\section{References}

1. Bray F, Ferlay I, Soerjomataram I, et al. Global cancer statistics 2018: GLOBOCAN estimates of incidence and mortality worldwide for 36 cancers in 185 countries. CA Cancer J Clin 2018;68:394-424.

2. $\mathrm{Xu}$ J, Stolk JA, Zhang $X$, et al. Identification of differentially expressed genes in human prostate cancer using subtraction and microarray. Cancer Res 2000;60:1677-1682.

3. Zheng SL, Chang B, Faith DA, et al. Sequence variants of alpha methylacyl-CoA racemase are associated with prostate cancer risk. Cancer Res 2002;62:6485-6488.

4. Freedland SJ, Humphreys EB, Mangold LA, et al. Risk of prostate cancer-specific mortality following biochemical recurrence after radical prostatectomy. JAMA 2005;294:433-439.

5. Shapovalova M, Davydova J, Henzler C, et al. Exploiting the Transcriptional specificity of the alpha-methylacyl-CoA racemase AMACR promoter for the molecular imaging of prostate cancer. Oncotarget 2018;9:36693-36704.

6. Ying Z, Feng L, Dongqing Ji, et al. Phase-regulated sensing mechanism of mos 2 based nanohybrids toward point-of-care prostate cancer diagnosis. Small 2020;16:e2000307. doi: 10.1002/smll.202000307.
7. Rubin MA, Bismar TA, Andrén O, et al. Decreased $\alpha$ - Methylacyl CoA racemace expression in localized prostate cancer is associated with an increased rate of biochemical recurrence and cancer-spesific death. Cancer Epidemiol Biomarkers Prev 2005;14:1424-1432.

8. Kars M, Gökmen E, Özman O, et al. Relationship between AMACR staining density of radical prostatectomy specimen and biochemical recurrence in patients with pathological stage T2a-b. Bull Urooncol 2020;19:38-41.

9. Rubin MA, Whittington R, Malkowicz SB, et al. Alpha-Methylacyl coenzyme $A$ racemase as a tissue biomarker for prostate cancer. JAMA 1998;280:969-974.

10. Box A, Alshalalfa M, Hegazy SA, et al. High alpha-methylacylCoA Racemase (AMACR) is associated with ERG expression and with adverse clinical outcome in patients with localized prostate cancer. Tumour Biol 2016;37:12287-12299. doi: 10.1007/ s13277-016-5075-1.

11. Luo J, Zha S, Gage RW, et al. Alpha-methylacyl-CoA racemase: a new molecular marker for prostate cancer. Cancer Res 2002;62:2220-2226.

12. de Souza MF, Kuasne H, Barros-Filho MC, et al. Circulating mRNA signature as a marker for high-risk prostate cancer. Carcinogenesis 2020;41:139-145.

13. Ji J, Chen $\mathrm{X}, \mathrm{Xu} \mathrm{Y}$, et al. Prostate cancer diagnosis using urine sediment analysis-based $\alpha$-methylacyl-coa racemase score: a singlecenter experience. Cancer Control 2019;26:1073274819887697. doi: $10.1177 / 1073274819887697$.

14. Henttu P, Liao SS, Vihko P. Androgens up-regulate the human prostate-specific antigen messenger ribonucleic acid (mRNA), but down-regulate the prostatic acid phosphatase mRNA in the LNCaP cell line. Endocrinology 1992;130:766-772.

15. Robles M], de Torres Mateos JA, and Soler-Rosello A. [Analysis of the serum concentration of prostate-specific antigen as a biological marker in the evolution of disseminated prostatic cancer]. Actas Urol Esp 1988;12:152-157.

16. Čapoun O, Soukup V, Kalousová M, et al. Diagnostic importance of selected protein serum markers in the primary diagnostics of prostate cancer. Urol Int 2015;95:429-435.

17. Yu YP, Tsung A, Liu S, et al. Detection of fusion transcripts in the serum samples of patients with hepatocellular carcinoma. Oncotarget 2019;10:3352-3360.

18. Etheridge T, Straus J, Ritter MA, et al. Semen AMACR protein as a novel method for detecting prostate cancer. Urol Oncol 2018;36:532.e1532.e7. doi: 10.1016/j.urolonc.2018.09.010. 